\title{
Polímeros Inteligentes em Dispositivos Biomédicos e Bio-separação
}

\author{
Ana Aguiar-Ricardo*
}

\begin{abstract}
Nos s últimos trinta anos tem-se assistido a uma mudança de paradigma importante: os cientistas têm conseguido sintetizar polímeros com capacidade de adquirir conformações controláveis por estímulos externos como temperatura, $\mathrm{pH}$, substâncias químicas específicas ou outros, e construir sistemas multifuncionais com interfaces capazes de responder também a estímulos externos, o que se pensava ser um exclusivo dos sistemas vivos. Estas superfícies inteligentes permitem a manipulação das propriedades dos materiais com o objetivo de promover ou impedir a adesão e a adsorção de células, a ligação de biomoléculas e, até, dirigir as funções celulares. Uma estratégia recente na investigação tem sido o design e construção de nano- ou macroestruturas inteligentes utilizando processos integrados e sustentáveis. Este artigo evidencia como o nosso grupo tem contribuído para resolver alguns problemas tecnológicos relevantes utilizando dióxido de carbono supercrítico: como preparar micropartículas de polímeros inteligentes com um fino controlo da morfologia 3D e das propriedades mecânicas? Como conferir a capacidade de resposta a estímulos exteriores a estruturas poliméricas $3 D$ ? Em cada um dos estudos descritos identificam-se as propriedades dos materiais que se conseguiram otimizar, as vantagens do ponto de vista de sustentabilidade das metodologias seguidas e as perspetivas de futuras aplicações nas áreas de libertação controlada de fármacos, engenharia de tecidos, biossensores e bio-separação.
\end{abstract}

\section{INTRODUÇÃO}

Designam-se por polímeros ativos ou inteligentes, macromoléculas que são capazes de alterar visivelmente a sua estrutura, ou determinada propriedade física ou química, de uma forma rápida e reversivel, como resposta a pequenas variações de estímulos externos, nomeadamente alterações de temperatura, $\mathrm{pH}$, concentração de um dado agente químico, etc. Estímulos como o pH, a força iónica ou agentes químicos, são designados por estímulos químicos, pois levam à alteração das interações a nível molecular entre as cadeias poliméricas, ou entre estas e o solvente em que se encontram. Os estímulos físicos como a temperatura, os campos elétrico e magnético e o stress mecânico afetam os níveis energéticos de diferentes tipos das moléculas, alterando as interações moleculares. Estas capacidades de resposta de muitos sistemas poliméricos têm aplicações amplas em libertação controlada de fármacos [1-4], em biotecnologia [5] e em cromatografia [6]. Existem inúmeros sistemas poliméricos que conseguem responder até a mais do que um estímulo.

Universidade Nova de Lisboa, Faculdade de Ciências e Tecnologia, Departamento de Química, Campus de Caparica, 2829-516 Caparica

E-mail: p19@fct.unl.pt
Os polímeros capazes de responder a variações de temperatura têm sido os mais estudados. Apresentam na sua estrutura um balanço fino entre as interações estabelecidas pelas partes de cadeia hidrofílica e hidrofóbica e, devido a pequenas variações de temperatura em torno de um valor crítico $\mathrm{T}$, colapsam ou expandem reajustando as interações hidrofóbicas e hidrofílicas entre as cadeias ou as interações hidrofílicas entre as cadeias e o meio aquoso. A maior parte das aplicações destes sistemas está relacionada com sistemas que apresentam uma temperatura crítica mínima de solução (LCST - lower critical solution temperature), isto é, considerando um diagrama temperatura-composição, a curva que delimita a região de imiscibilidade apresenta um mínimo. O polímero mais comum que apresenta uma LCST em solução aquosa é o poli(N-isopropilacrilamida) (PNIPAAm), o qual sofre uma variação brusca de volume resultante da passagem de uma estrutura expandida, com prevalência de interações por pontes de hidrogénio entre as cadeias do polímero e as moléculas de água, para uma estrutura colapsada com prevalência de interações hidrofóbicas entre os grupos isopropilo das cadeias poliméricas, expelindo a água do interior da cadeia colapsada. O PNIPAAm tem uma LCST de cerca de $32^{\circ} \mathrm{C}$, que é próxima da temperatura fisiológica, apresentando assim solubilidade em água a temperaturas inferiores a $32^{\circ} \mathrm{C}$ e precipitando a temperaturas superiores. Para se prepararem estruturas tridimensionais com capacidade de absorver ou expelir grandes quantidades de água por manipulação de um dado estímulo a partir de polímeros solúveis utilizam-se agentes reticulantes. Obtêm-se assim hidrogéis. Quando um hidrogel está desidratado, as cadeias do polímero encontram-se colapsadas, dificultando os fenómenos de difusão através da estrutura; pelo contrário, quando o hidrogel incha e atinge o valor de inchamento de equilíbrio, atinge o tamanho de malha de rede máximo ( $\xi$, "mesh size") e os fenómenos que dependem de mecanismos difusionais apresentam valores máximos (Figura 1).

A LCST de um polímero cujas propriedades podem ser manipuladas por

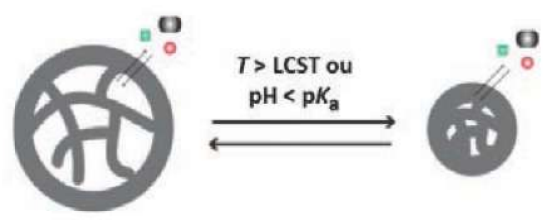

Figura 1 - Esquema ilustrativo da variação de volume que se observa num polímero que responde à temperatura e/ou $\mathrm{pH}$. Também se mostra o impacto do inchamento do

hidrogel na permeação e libertação de outras moléculas. Adaptado de [7] 
variação de temperatura é influenciada pelas unidades hidrofílicas e hidrofóbicas que estão presentes nas suas cadeias. Em geral, para aumentar a LCST, por exemplo, o PNIPAAm pode ser copolimerizado com uma fracção de monómeros mais hidrofílicos como o ácido metil metacrílico [8], enquanto que para baixar a LCST se pode copolimerizar com um monómero menos hidrofílico como o 2-hidroxietilmetacrilato (HEMA). Na Figura 2 estão representados alguns dos polímeros mais estudados que apresentam LCST.

Outros polímeros com muitas aplicações são os que respondem ao pH. Estes polímeros são polielectrólitos que contêm na sua estrutura grupos funcionais ácidos (poliácidos) ou bási$\cos$ (polibases) fracos e que portanto cedem ou recebem protões como resposta a variações de $\mathrm{pH}$ do meio. À medida que $\circ \mathrm{pH}$ se altera, o grau de ionização dos grupos funcionais ácidos ou básicos muda drasticamente a um dado $\mathrm{pH}$ que se designa por $\mathrm{p} K_{\mathrm{a}}$. Como resultado da carga que se gera ao longo da cadeia do polielectrólito há um aumento das forças repulsivas que só podem ser neutralizadas com contra-iões que por isso migram do seio da solução para a estrutura polimérica, com consequente aumento do volume hidrodinâmico do polímero. A gama de $\mathrm{pHs}$ em que se dá a transição pode ser estabelecida selecionando os grupos funcionais com um $p K_{\mathrm{a}}$ que esteja no intervalo pretendido, em função da aplicação pretendida ou incorporando grupos hidrofóbicos na cadeia polimérica. Quando os grupos funcionais não estão ionizados, as forças repulsivas entre os grupos carregados dentro do polímero não existem e dominam as forças hidrofóbicas. Nesta situação, o polímero adquire uma estrutura mais compacta e apresenta uma transição mais brusca. A hidrofobicidade da cadeia pode ser controlada, copolimerizando os monómeros ionizáveis hidrofílicos com outros monómeros mais hidrofóbicos como o 2-hidroxietil metacrilato, o metil metacrilato e o anidrido maleico.

Os poliácidos apresentam a estrutura mais compactada a menores $\mathrm{pHs}$, pois nessa situação os grupos carboxílicos estão protonados. Quando o $\mathrm{pH}$ aumenta, o polímero fica car-

(a)

(b)<smiles>CC(C)NC(=O)C(CC(C)(C)C)C(C)(C)C</smiles><smiles>CCN(CC)C(=O)C(C(C)(C)C)C(C)(C)C</smiles>

(c)<smiles>CN(C)CCOC(=O)C(C)(CC(C)(C)C)C(F)(F)F</smiles>

(d)

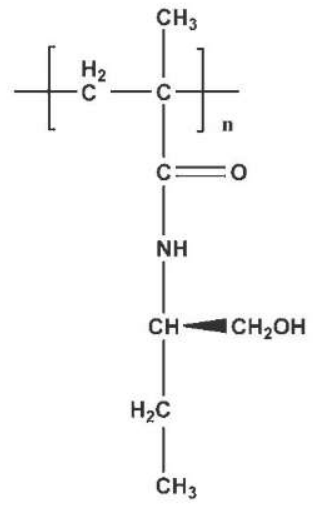

Figura 2 - Estruturas dos polímeros que apresentam LCST próxima da temperatura ambiente. (a) PNIPAAm, LCST $32^{\circ} \mathrm{C}$; (b) poli(N, $\mathrm{N}^{\prime}$-dietilacrilamida) (PDEAAm), LCST $26-35^{\circ} \mathrm{C}$;

(c) poli(metacrilato de 2-dimetilaminoetilo) (PDMAEMA), LCST $50^{\circ} \mathrm{C}$; (d) poli( $\mathrm{N}$-(L)-(hidroximetil) propilmetacrilamida) LCST $30^{\circ} \mathrm{C}$

regado negativamente e incha; pelo contrário, as polibases aumentam o grau de ionização à medida que o pH diminui. Alguns dos polímeros mais representativos que respondem a variações de $\mathrm{pH}$ estão identificados na Figura 3. Tipicamente são polímeros com grupos carboxílicos como o poli(ácido metacrílico) (Figura 3(b)), ou também podem ser derivados de poli(sulfonamidas). Alguns exemplos de polímeros policatiónicos são a poli(lisina) ( $\mathrm{PL})$, poli(etilenoimina) (PEI) e o quitosano (Figuras 3(e), 3(f) e $3(g))$.

O estudo de sistemas poliméricos ativos e das suas propriedades mais relevantes, em aplicações em dispositivos de libertação controlada, em tecnologias de tecidos ou em bio-separação, tem sempre de ser acompanhado do estudo do efeito de parâmetros termodinâmicos (ex. temperatura, $\mathrm{pH}$, força iónica) na evolução do espaço do diagrama de fases em que ocorrem as transições de fases e em que se definem os limites de imiscibilidade.
Apesar da crescente atividade científica com o objetivo de desenvolver novos polímeros inteligentes e novas metodologias sintéticas, ou para conseguir sistemas ativos tridimensionais com importantes aplicações nas mais variadas áreas, só nas últimas décadas é que chegaram ao mercado alguns dos protótipos desenvolvidos. O primeiro produto "Smart-Gel" foi comercializado em 1996 pela Gel Sciences/GelMed (Bedford, Massachusetts), tratando-se de uma palmilha anatómica formada por um gel viscoelástico, muito maleável à temperatura ambiente mas que, quando em contacto com o pé, se torna mais firme adaptando o sapato ao pé de cada um. Mais recentemente, uma companhia japonesa, CellSeed (www. cellseed.com/company-e/index.html), lançou para o mercado placas de 96 poços e outros dispositivos para o crescimento de tecidos celulares com a possibilidade de fazer a recolha do tecido celular de uma forma mais eficaz, apenas por variação da temperatura, conseguindo assim substituir a 
utilização de tratamentos enzimáticos que danificam a membrana basal. Nos últimos anos tem havido inúmeros esforços no sentido de melhorar as metodologias de síntese e processamento de polímeros utilizando processos mais sustentáveis. Em particular, a utilização de tecnologia supercrítica, nomeadamente, dióxido de carbono supercrítico $\left(\mathrm{scCO}_{2}\right)$ como meio reacional para polimerizações ou como meio de processamento de materiais. Processos envolvendo $\mathrm{scCO}_{2}$ apresentam em geral inúmeras vantagens, comparativamente aos processos convencionais, pois possibilitam em muitos casos a redução de consumo de solventes orgânicos e de água, a minimização de processos de limpeza e tratamentos de águas e, ainda, uma maior integração de processos. Há vários exemplos na literatura [9]

A utilização de $\mathrm{CO}_{2}$ supercrítico para preparar hidrogéis apresenta vantagens que ainda se tornam mais interessantes quando os materiais se destinam a aplicações biomédicas, dada a facilidade com que se eliminam quaisquer quantidades vestigiárias de contaminantes e se obtêm materiais completamente limpos. Desenvolvimentos recentes de novos processos que combinam a tecnologia supercrítica com outras tecnologias têm permitido desenhar e obter dispositivos que pelos processos convencionais não seriam possíveis de obter ou, pelo menos, não teriam um desempenho tão bom em determinadas aplicações. Apresentam-se de seguida alguns desses avanços, realçando em cada estudo as propriedades que se conseguiram otimizar, as vantagens do ponto de vista de sustentabilidade das metodologias seguidas e as perspetivas de futuras aplicações nas áreas de libertação controlada de fármacos, engenharia de tecidos, biossensores e bio-separação.

Polímeros inteligentes e $\mathrm{CO}_{2}$ suPERCRÍTICO

O PNIPAAm foi produzido pela primeira vez em $\mathrm{CO}_{2}$ supercrítico por Temtem et al. [10] sem que fosse necessária a adição de quaisquer solventes orgânicos ou aditivos. Para estudar e otimizar a síntese de PNIPAAm na forma de hidrogel, a síntese foi feita com diferentes razões de metilbisacrilamida (MBAm), tendo-se verificado que a capacidade de resposta à temperatura diminuía significativamente para frações mássicas de MBAm su- periores a $2,4 \%$. Nessas condições é possível obter partículas com um diâmetro médio que varia entre 2 a $6 \mu \mathrm{m}$, diminuindo com o aumento do grau de reticulação e apresentando também maior ou menor agregação em função do grau de reticulação e das condições operacionais da síntese. Neste estudo, a polimerização deu-se por precipitação em $\mathrm{CO}_{2}$ supercrítico. Existem poucos relatos de trabalhos de síntese de micropartículas poliméricas de morfologia controlada por polimerização por precipitação, porque esta estratégia só é aplicável a monómeros com solubilidade apreciável em $\mathrm{CO}_{2}$ supercrítico. Para conseguir polimerizar eficazmente monómeros menos solúveis há que realizar a polimerização por dispersão, por emulsão ou suspensão, utilizando estabilizadores, isto é, moléculas anfifílicas capazes de formar agregados na fase supercrítica, conseguindo assim estabilizar os monómeros e os oligómeros em crescimento até que estes atinjam um determinado peso molecular. Como em geral os polímeros não são solúveis em $\mathrm{CO}_{2}$ supercrítico, ao atingirem um dado peso molecular as cadeias poliméricas deixam de ser suportadas pelos agregados formados pelos estabilizadores e precipitam no fundo (a)

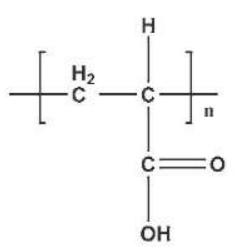

(e) (b)<smiles>CCC(C)(C)C(=O)O</smiles>

(c)

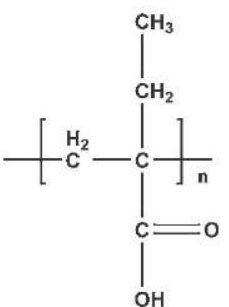

(d)

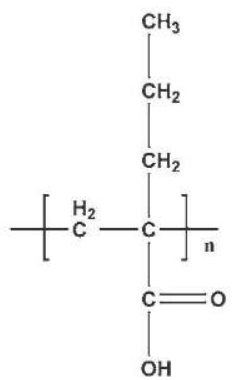

(g)<smiles>CC(C)(C)NCCCC[C@H](N)C(=O)C(C)(C)C</smiles>

(f)<smiles>CC(C)(C)CCNC(C)(C)C</smiles>

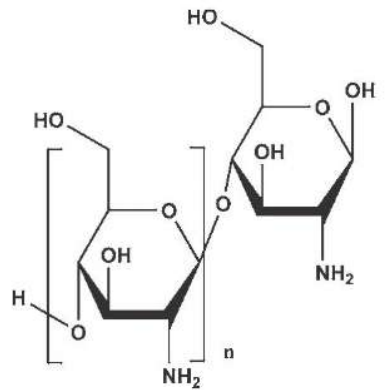

Figura 3 - Alguns dos polímeros mais representativos que respondem ao $\mathrm{pH}$; (a) poli(ácido acrílico) (PAAc), (b) poli(ácido metacrílico) (PMAAc), (c) poli(2-etilo ácido acrílico) (PEAAc), (d) poli(2-propilo ácido acrílico) (PPAAc), (e) poli(lisina) (PL), (f) poli(etilenoimina) (PEI) e (g) quitosano (CHT) 


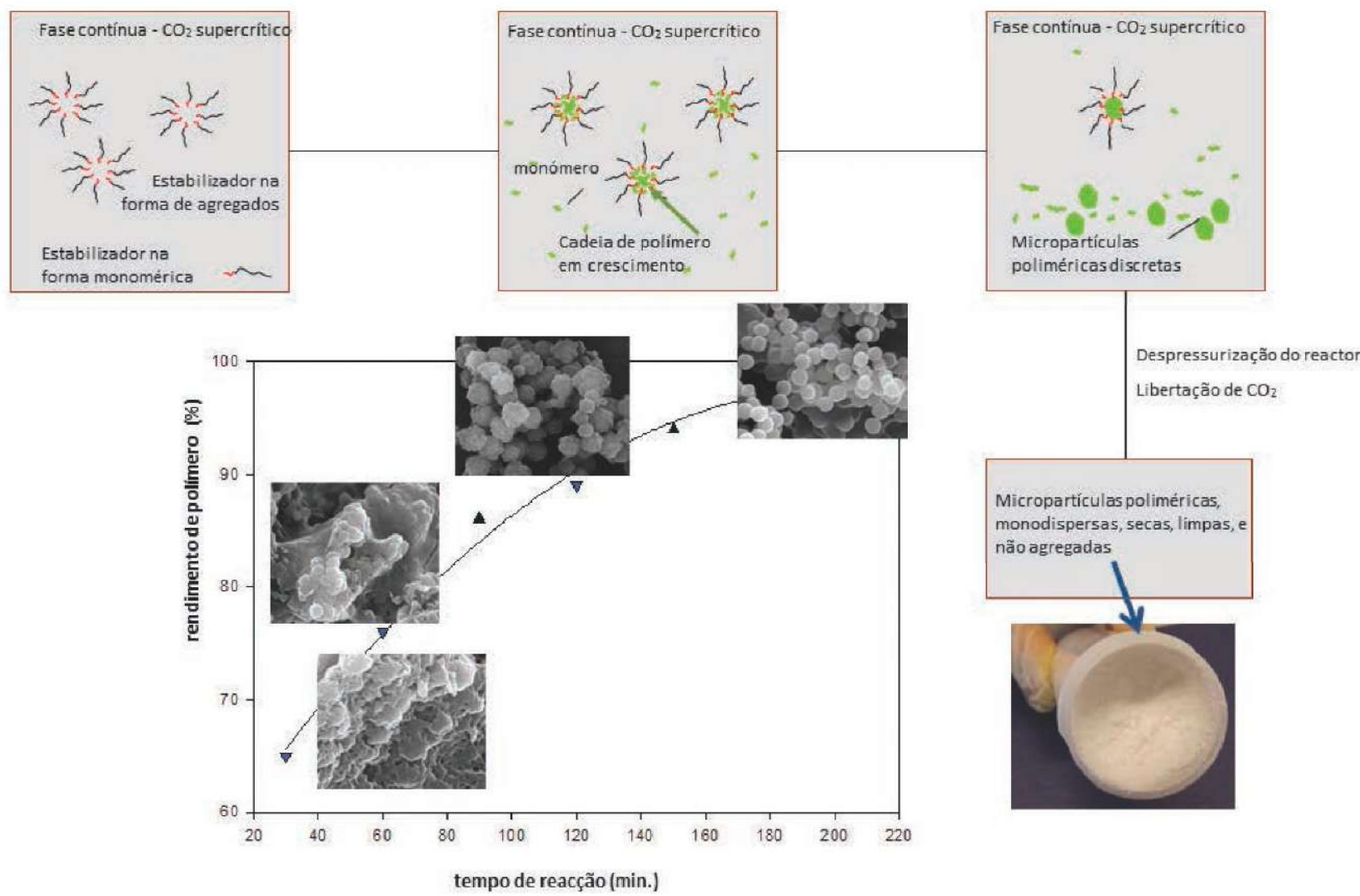

Figura 4 - Esquema demonstrativo do mecanismo da polimerização por dispersão em $\mathrm{CO}$, supercrítico. Apresentam-se também imagens de microscopia de varrimento eletrónica de polimeros obtidos a diferentes tempos de reação para melhor visualização da evolução da morfologia dos agregados. Adaptado de [11]

do reator na forma de partículas esféricas livres e bastante monodispersas (ver Figura 4).

Como a precipitação se dá para as partículas poliméricas que atingem um certo peso molecular, os polímeros sintetizados em condições supercríticas apresentam baixa polidispersividade. A maior parte dos estabilizadores de polimerização em $\mathrm{CO}_{2}$ supercrítico têm uma cadeia alquílica que se pode ligar covalentemente à cadeia de polímero em crescimento ou ficar "aprisionada" nas cadeias em crescimento, contaminando assim o produto final. Contudo, o Krytox®, que é um ácido carboxílico perfluorado disponível no mercado, tem-se mostrado extremamente eficaz na estabilização de polimerizações radicalares de diferentes monómeros, através do estabelecimento de interações reversiveis por pontes de hidrogénio com as cadeias em crescimento; dada a sua elevada solubilidade em $\mathrm{CO}_{2}$ supercrítico, no final da reação, o Krytox (B) é completamente removido do polímero por simples lavagem com $\mathrm{CO}_{2}$ supercrítico $[11,12]$.
Após o trabalho pioneiro de Temtem et al., a polimerização do NIPAAm utilizando diferentes reticulantes e a sua copolimerização com diferentes monómeros em $\mathrm{CO}_{2}$ supercrítico foi estudada de uma forma sistemática com o objetivo de preparar microgéis com morfologias e propriedades reológicas muito bem definidas. Utilizaram-se reticulantes com hidrofilicidade crescente: MBAm < DEGDMA < GDMA, mas sempre com a mesma percentagem mássica $(1,2 \%)$ para assegurar a capacidade de responder a variações de temperatura. Prepararam-se microgéis por copolimerização de NIPAAm com acrilato de poli(etilenoglicol) (PEGa, $M_{n}=375$ ) e com ácido metacrílico (MAA), variando as composições das misturas de monómeros iniciais. Neste estudo, Costa et al. [13] visava a produção de partículas de hidrogéis biocompatíveis que funcionassem como "pseudocélulas" sintéticas em plataformas de monitorização dos microambientes em culturas de tecidos celulares. Enquanto que para sistemas de libertação controlada de fármacos interessa muitas vezes preparar nanopartículas, para uma aplicação de bio-detecção ou biomonitorização, o tamanho médio típico do microgel, na forma das "pseudocélulas", terá de ser superior a 3-4 $\mu \mathrm{m}$, para evitar que seja incorporado na célula, e também ter propriedades mecânicas bem definidas. O estudo conduziu à conclusão de que os microgéis com maior potencial para o desenvolvimento de uma plataforma de monitorização eram os microgéis do copolímero poli(NIPAAm-co-MAA) (aqui designado por P(NIPAAm-co-MAA)), preparados com $10 \%$ de MAA (razão mássica relativa ao NIPAAm). A copolimerização com MAA conferiu aos microgéis, quer grupos funcionais disponíveis para posterior funcionalização, quer capacidade de resposta ao $\mathrm{pH}$, aumentando assim a gama de aplicações destes microgéis [14, 15]. Quando imersas em ambiente fisiológico $(\mathrm{pH}$, temperatura e força iónica) as partículas apresentavam cerca de $11 \mu \mathrm{m}$. Um resultado muito interessante foi que estas partículas apresentaram também uma malha suficientemente larga para permitir a permeação de metaloproteases (como MMP-9) através delas. Por último, os 
estudos realizados para avaliação das propriedades reológicas e da biocompatibilidade destas partículas mostraram que estas tinham valores de viscoelasticidade conformes com os pretendidos e que não apresentavam qualquer efeito tóxico sobre culturas de fibroblastos [13]

Os microgéis de P(NIPAAm-co-MAA) apresentam uma capacidade de inchamento que depende drasticamente da temperatura e do $\mathrm{pH}$ do meio, embora com ordens de grandeza que variam consoante a composição do copolímero relativamente a cada monómero. Esta capacidade de inchamento pode ser usada para controlar a libertação de fármacos incorporados na matriz do microgel. Assim, impregnou-se 5-fluorouracil (5-FU) em microgéis com diferentes composições relativas dos dois monómeros NIPAAm e MAA para avaliar o desempenho dos microgéis sintetizados como sistemas pulsáteis de libertação de fármacos. Os perfis farmacocinéticos de libertação do 5-FU em função da temperatura e do $\mathrm{pH}$ do meio estão representados na Figura 5. É evidente a resposta dos microgéis aos dois estímulos, temperatura e $\mathrm{pH} . \mathrm{A} 37^{\circ} \mathrm{C}$, o microgel apresenta uma estrutura colapsada e por isso só se observa uma pequena libertação de fármaco enquanto que à temperatura mais baixa, em que se tem $\mathrm{T}$ menor que LCST, o microgel passa para uma estrutura expandida e quase todo o 5-FU se liberta imediatamente. As diferenças nas quantidades de 5-FU libertados ( 2 mg 5-FU/g de microgel, quando se têm partículas de P(NIPAAm-Co-MAA) 60:40 e 4 mg 5 -FU/g de microgel, quando se usam partículas de P(NIPAAm) são devidas às diferentes cargas electroestáticas predominantes no 5-FU e no microgel. O estudo de libertação in vitro em função do $\mathrm{pH}$ mostra claramente o efeito de pulso que se consegue por manipulação do $\mathrm{pH}$.

\section{REVEstimento dE EstRutuRAS POROSAS}

O desenvolvimento de membranas ou outros dispositivos 3D com capacidade de resposta a estímulos externos pode ser conseguido utilizando metodologias diferentes: (i) uma consiste na sintese dos materiais inteligentes (polímeros ou copolímeros) seguida do seu processamento por forma a obter as membranas, "scaffolds" ou outros dispositivos; (ii) outra consiste em modificar por algum processo os materiais 3D ou membranas por forma a incorporar os polímeros inteligentes. Contudo, neste artigo focaremos apenas os trabalhos em que o desenvolvimento de dispositivos poliméricos inteligentes se baseou em metodologias assistidas por $\mathrm{CO}_{2}$ supercrítico na síntese ou no processamento dos materiais, numa tentativa de desenvolver e aplicar processos mais sustentáveis que envolvem menos solventes orgânicos e menos passos de purificação, limpeza e secagem.

Apesar da motivação inicial do desenvolvimento de sistemas poliméricos inteligentes ter sido a de administração de moléculas terapêuticas, muitas outras aplicações têm vindo a surgir em áreas como engenharia de tecidos e medicina regenerativa, bio-separação e desenvolvimento de sistemas de microfluidica. Quando se têm em vista aplicações biomédicas é extremamente importante evitar o uso e manipulação de quaisquer solventes orgânicos potencialmente tóxicos que se possam acumular nos materiais durante os passos de síntese ou processamento. A possibilidade de sintetizar de uma forma tão eficaz e tão controlada diferentes polímeros e copolímeros em $\mathrm{CO}_{2}$ supercrítico abriu uma janela de oportunidades para o desenvolvimento destes materiais inteligentes. Como $\circ \mathrm{CO}_{2}$ supercrítico consegue solubilizar quantidades apreciáveis de monómeros do tipo acrilato, tais como o NIPAAm e o $\mathrm{N}, \mathrm{N}$ '-dietilacrilamida (DEAAm), entre outros, e tem elevadas difusividades, consegue transportar os monómeros, impregnando diferentes estruturas porosas, e produzir in situ os correspondentes polímeros, revestindo os materiais com os produtos da reação. A prova deste conceito foi feita realizando a polimerização do NIPAAm sobre a matriz de "scaffolds" de quitosano previamente preparados por liofilização [10]. Numa das imagens de microscopia eletrónica de varrimento incluída na Figura 6 vê-se a morfologia típica de "scaffolds" de quitosano impregnados com PNIPAAm. Estes "scaffolds" mostraram-se com capacidade de resposta a variações de temperatura, e também ao $\mathrm{pH}$, devido às propriedades intrínsecas à matriz de quitosano, sem que a metodologia aplicada comprometesse, quer a porosidade, quer a biodegradabilidade desses mesmos "scaffolds". Num outro estudo, $\circ \mathrm{CO}_{2}$ supercrítico foi também aplicado primeiro para a reticu- (a)

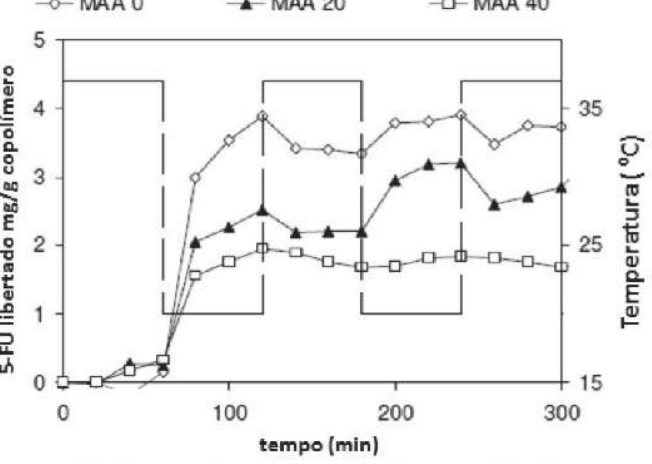

(b)

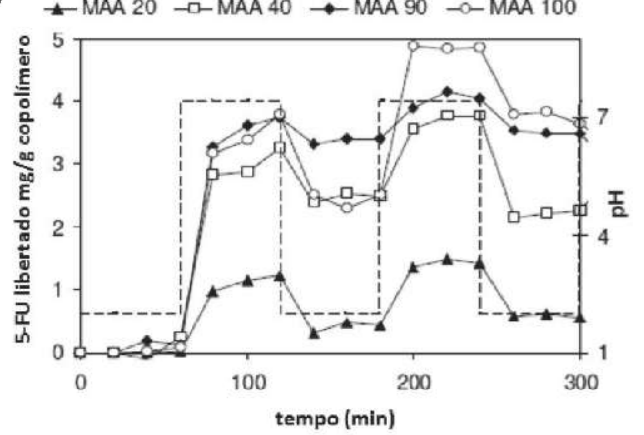

Figura 5-Perfis farmacocinéticos de libertação do 5-fluorouracil a partir de microgéis de $\mathrm{P}$ (NIPAAm-co-MAA) a diferentes temperaturas e $\mathrm{pH}$. Adaptado de [16] 
lação com glutaraldeído de "scaffolds" de quitosano e colagénio (CHT-CLG "scaffolds") e de seguida para polimerizar in situ DEAAm com a mesma metodologia já descrita [17]

Para explorar as potencialidades desta metodologia de revestimento de estruturas porosas poliméricas utilizando $\mathrm{CO}_{2}$ supercrítico realizaram-se outros estudos envolvendo matrizes de diferentes materiais. Num dos estudos, revestiram-se membranas de poli(sulfona) (PSF) e outras preparadas a partir de compósitos de PSF com poliacrilonitrilo (PAN), com dois hidrogéis diferentes, PNIPAAm e PDEAAm. Num primeiro passo, prepararam-se as membranas porosas de PSF por inversão de fases induzida por $\mathrm{CO}_{2}$ supercrítico [18]; de seguida, as membranas de PSF de morfologia bem definida foram revestidas/impregnadas com PNIPAAm por polimerização in situ. Para preservar a integridade do revestimento de PNIPAAm produzido, realizou-se a síntese na presença de agente reticulante, MBAm. Assim, o sistema pode inchar ou colapsar em meio aquoso, isto é, estender as suas cadeias ou colapsá- -las e, consequentemente, abrir ou fechar os poros da matriz sem que o PNIPAAm se dissolva. Este mecanismo reversível de distensão e contração das cadeias poliméricas traduz-se num sistema regulador "on-off" de controlo da permeabilidade da membrana. Quando a membrana está a uma temperatura inferior à LCST, só um pequeníssimo fluxo de água pode atravessar a membrana (permeabilidade à água $\sim 0 \mathrm{~L} \mathrm{~m}^{-2} \mathrm{~h}^{-1}$ bar $^{-1}$ ), pelo contrário, quando a temperatura é maior que a LCST, obtém-se um valor de permeabilidade à água $>300 \mathrm{Lm}^{-2} \mathrm{~h}^{-1} \mathrm{bar}^{-1}$. Pelo que nos é dado a saber, este enorme e repentino aumento de permeabilidade é a maior razão on-off reportada na literatura para permeabilidade à água em membranas inteligentes [19]. Isto significa que, com a densidade superficial que o PNIPAAm adquiriu à superfície dos poros e com o peso molecular com que foi sintetizado neste trabalho, as cadeias hidrofílicas do PNIPAAm adquirem uma conformação tão distendida abaixo de $32^{\circ} \mathrm{C}$ que obstruem de uma forma notável os poros da membrana, reduzindo o seu tamanho efetivo. Pelo contrário, acima da LCST, as interações entre (a)

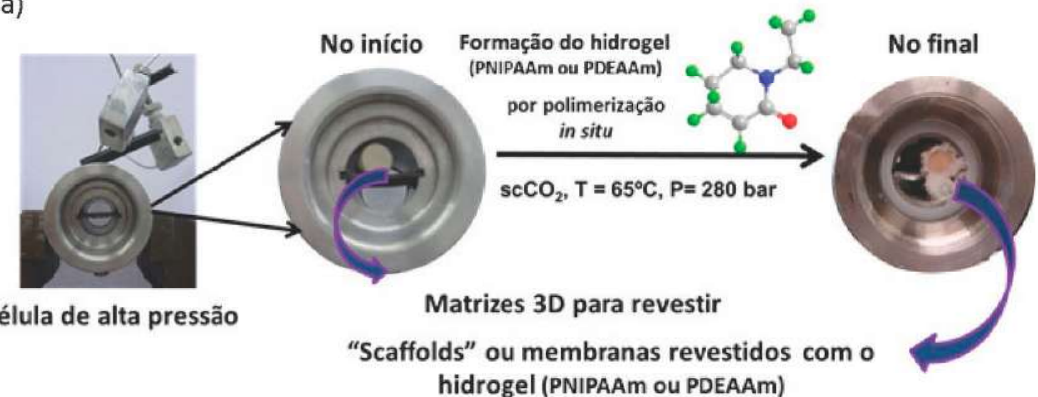
hidrogel (PNIPAAm ou PDEAAm)

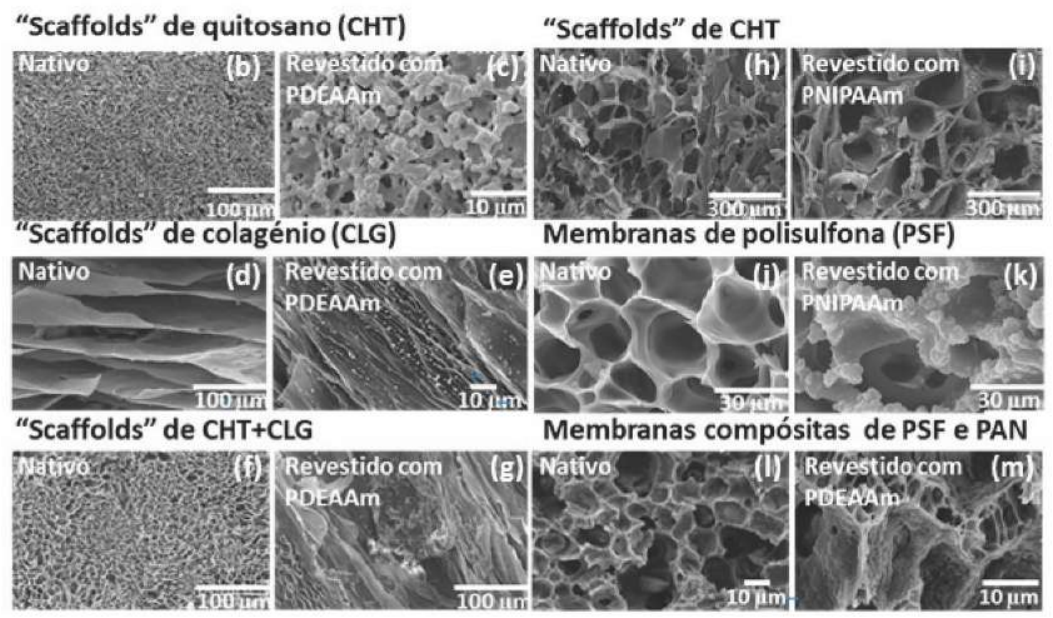

Figura 6 - (a) Esquema da metodologia para revestimento de diversas matrizes poliméricas com diferentes hidrogéis em $\mathrm{CO}_{2}$ supercrítico. (b) a $(\mathrm{m})$ imagens de microscopia eletrónica de varrimento de scaffolds e membranas antes e após o revestimento com os hidrogéis as cadeias do PNIPAAm tornam-se predominantemente hidrofóbicas com rompimento das pontes de hidrogénio estabelecidas com a água; as cadeias poliméricas assumem então uma conformação colapsada, os poros da matriz suporte ficam desimpedidos e permitem assim um maior fluxo de água como se esquematiza na Figura 7 .

No caso do estudo de membranas compósitas de poli(sulfona) e poliacrilonitrilo (membranas PSF/PAN), os autores verificaram que variando a razão de PAN nas soluções de polímero de partida, conseguiam controlar a morfologia da estrutura porosa em termos de tamanho médio de poro, porosidade global da membrana e as suas propriedades mecânicas, otimizando assim as propriedades da matriz de suporte para posterior revestimento com PNIPAAm e PDEAAm [20]. Após o revestimento das membranas com estes dois polímeros que têm capacidade de resposta à temperatura, as membranas apresentaram propriedades completamente díspares. Enquanto as membranas revestidas com PDEAAm apresentaram permeabilidades reguláveis por variação de temperatura e boa eficiência em processos de bio-separação, evidenciada nos ensaios de separação de duas proteínas modelo de tamanhos diferentes, albumina de soro bovino (BSA) e lisozima (LYS), as membranas PSF/PAN revestidas com PNIPAAm não conseguiram adquirir capacidade de resposta a variações de temperatura, apresentando assim perfis de permeação de proteínas muito semeIhantes abaixo e acima da LCST do PNIPAAm.

Como se demonstrou através dos exemplos anteriores, o desempenho de uma dada membrana, que foi desenhada para responder a estímulos de temperatura, resulta da combinação de diferentes fatores estruturais e químicos. O resultado da interação entre as características morfológicas e composição química das matrizes suportes com os revestimentos de hidrogéis que respondem à temperatura pode assim conduzir a membranas com boa capacidade de resposta à temperatura (ex. membrana de PSF revestida com PNIPAAm) ou, pelo contrário, produzir outras (ex. mem- 
brana de PSF-PAN revestida com PNIPAAm) em que o hidrogel deixa de conseguir responder (Figura 8 ).

Uma das metodologias mais usada para funcionalizar ou revestir superfícies com polímeros inteligentes consiste em proceder ao enxerto destes polímeros por ligações covalentes às superfícies suporte. Habitualmente, o procedimento começa com a ativação da superfície dos suportes com espécies radicalares, a partir das quais se inicia a polimerização por adição de monómero. Um processo simples para ativar as superfícies é irradiá-las com um gás na forma de plasma (ex. plasma de árgon) e assim criar os iniciadores radicalares à superfície. Nas técnicas mais convencionais de "enxerto" ("grafting"), os materiais são ativados radicalarmente e imersos em solução de monómero. No final do processo, há que proceder a inúmeros passos de lavagem, nuns casos com solventes orgânicos, noutros com água, para eliminar completamente quaisquer vestígios de monómero retido nos suportes. As superfícies revestidas utilizando esta metodologia apresentam em geral alguma irregularidade na morfologia da camada de hidrogel depositada, quer em termos de espessura da camada, quer em termos de densidade de polímero enxertado [21]. Esta limitação pode ser impeditiva do uso desta metodologia para aplicações em que há necessidade de um grande controlo da nano- e microestrutura das superfícies. Recentemente, o nosso grupo desenvolveu uma metodologia mais limpa combinando a ativação por plasma com a polimerização em $\mathrm{CO}_{2}$ supercrítico. Esta estratégia integrada foi testada em diferentes materiais e em dispositivos com diferentes geometrias, permitindo a obtenção dos materiais revestidos com camadas de hidrogéis com morfologia controlada, sem vestígios de quaisquer reagentes e prontos para usar sem que houvesse necessidade de passos suplementares de purificação. Recentemente, Barroso et al. [22] demonstraram a aplicabilidade e a eficiência desta estratégia, quer para revestir membranas porosas de PSF/PAN com PDEAAm, quer para revestir partículas aqui designadas por micro-suportes, cytopore 2 (CYT2) e cytodex 3 (CYT3) com
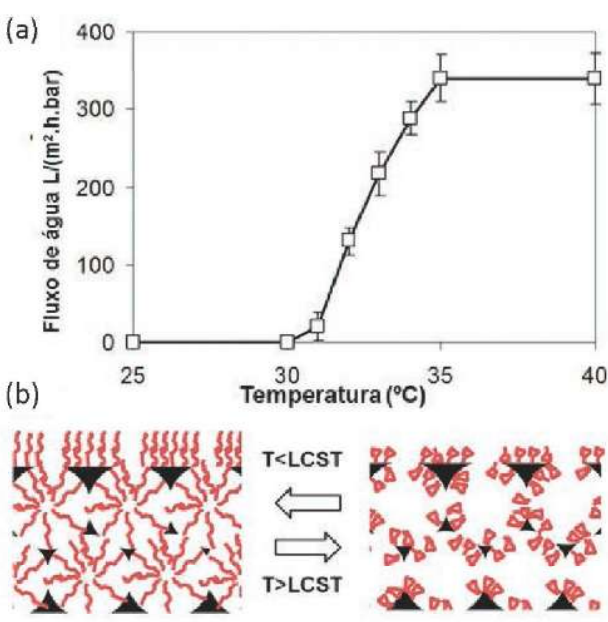

Figura 7 - (a) Variação do fluxo de água através de uma membrana de poli(sulfona) (PSF) revestida com PNIPAAm. (b) representação esquemática do mecanismo de controlo de tamanho de poro por variação de temperatura: a $T<\mathrm{LCST}$, as cadeias poliméricas estão expandidas, os poros ficam obstruídos, diminuindo a permeabilidade; a $T>\mathrm{LCST}$, as cadeias poliméricas estão colapsadas, os poros abrem, aumentando a permeabilidade. Adaptado de [18]. Copyright (2009) Royal Society of Chemistry (a)
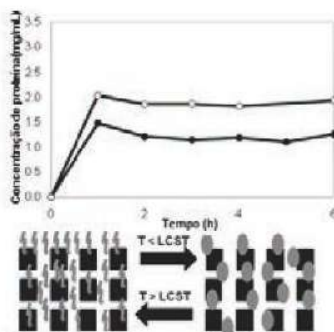
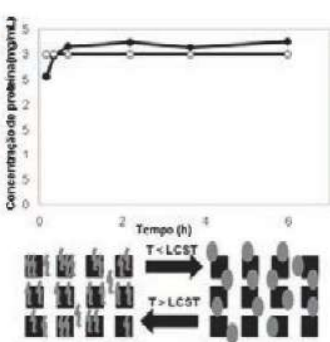

(c)

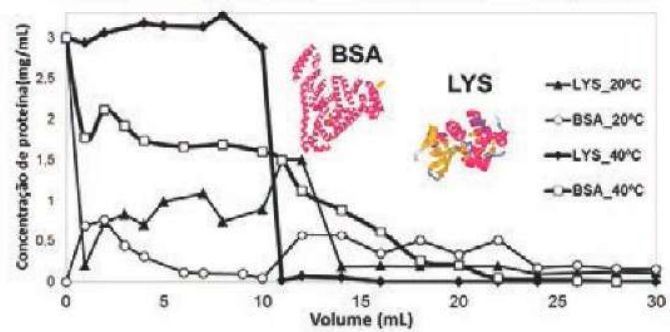

Figura 8 - Perfis de permeação da albumina do soro bovino (BSA) a $20 \circ \mathrm{C}\left(\bullet\right.$ ) e a $40{ }^{\circ} \mathrm{C}(\mathrm{o})$ : através de membranas de (a) poli(sulfona) (PSF) e de (b) PSF/PAN (90:10) revestidas com PNIPAAm obtido por polimerização in situ em $\mathrm{CO}_{2}$ supercrítico; (c) perfis de permeação da albumina do soro bovino (BSA) e da lisozima (LYS) através de membranas de PSF/PAN (90:10) revestidas com PDEAAm: permeação de BSA a $20^{\circ} \mathrm{C}(\bullet)$, permeação de BSA a $400^{\circ} \mathrm{C}(\mathrm{o})$, permeação de LYS a $20^{\circ} \mathrm{C}$

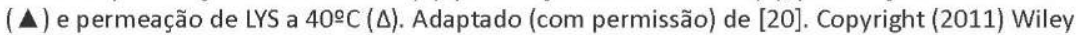
Online Library

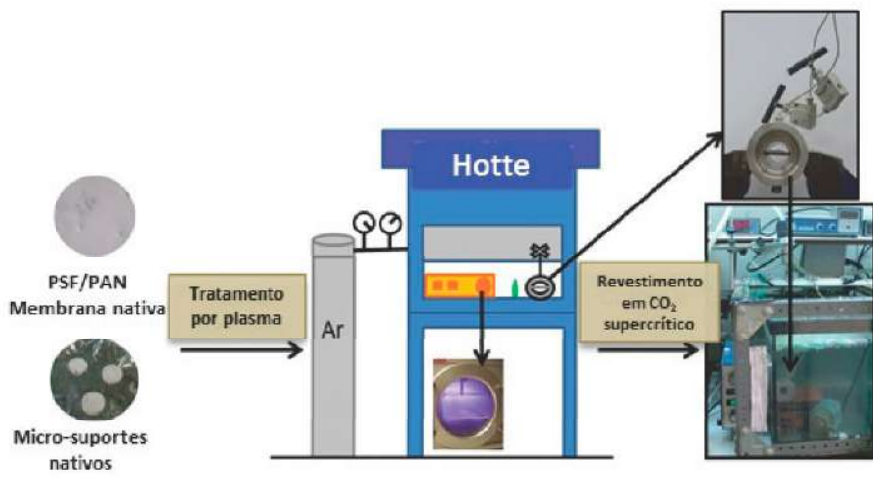

(a)

(b)

(c)

Figura 9 - (a) Matrizes poliméricas. (b) ativação das matrizes poliméricas por tratamento com plasma e introdução das matrizes ativadas na célula de alta pressão para posterior polimerização em $\mathrm{CO}_{2}$ supercrítico. (c) instalação de alta pressão usada para realizar a polimerização dos hidrogéis sobre as matrizes ativadas. Adaptado (com permissão) de [22]. Copyright (2012) American Chemical Society 
PNIPAAm, micro-suportes estes utilizados em bio-reatores aquando da expansão ex-vivo de células. O procedimento aplicado tem dois passos essenciais: primeiro, a formação de radicais à superfície da matriz suporte por tratamento com plasma (ex. tratamento com plasma de árgon) e, segundo, a sintese do hidrogel em $\mathrm{CO}_{2}$ supercrítico.

A polimerização in situ do NIPAAm sobre CYT2 e CYT3 ativados e do DEAAm a partir das membranas de PSF/PAN ativadas foi feita seguindo o procedimento habitual já descrito em artigos anteriores [10, 18], mas com as seguintes modificações [22]: i) os hidrogéis foram sintetizados na instalação de alta pressão usando uma célula com um separador central, a $37^{\circ} \mathrm{C}$ e $20 \mathrm{MPa}$, assegurando-se nestas condições de alta densidade um elevado poder solvente para $\circ \mathrm{CO}_{2}$; ii) sem a adição de iniciador radicalar, pois as matrizes já se encontravam previamente ativadas por tratamento com plasma; iii) todos os materiais foram manipulados numa hotte com atmosfera inerte controlada, para minimizar quaisquer reações secundárias com o oxigénio do ar e formação de peróxidos durante a transferência dos materiais da câmara do plasma para a célula de alta pressão (ver Figura 9). No final da reação, os materiais são lavados com $\mathrm{CO}_{2}$ fresco para assegurar a remoção de qualquer monómero que não tenha reagido. Quer o PNIPAAm, quer o PDEAAm foram sintetizados sem qualquer reticulante para maximizar a capacidade de resposta a estímulos de temperatura [10].

Como já foi dito, o cytopore 2 (CYT2) e o cytodex 3 (CYT3) são exemplos de microesferas utilizadas para a expansão de células que precisam de pontos de aderência para a sua proliferação $[23,24]$. No final, as células expandidas são recuperadas por um processo de tripsinização, que consiste na utilização de uma enzima proteolítica, designada por tripsina, para lisar (quebrar) o citoesqueleto celular que fornece sustentação às células para aderirem aos suportes em que cresceram. Esta técnica de recolha das células leva a consideráveis perdas das células viáveis. Há necessidade de desenvolver estratégias alternativas à tripsinização que consigam promover a desadsorção das células aderentes de uma forma menos agressiva, como seja a utilização de revestimentos inteligentes que consigam regular a desadsorção das células. A expansão ex-vivo de células estaminais mesenquimatosas é feita em reatores a $37^{\circ} \mathrm{C}$, que é superior à LCST do PNIPAAm (LCST $32^{\circ} \mathrm{C}$ ); se a temperatura descer abaixo da LCST, as cadeias do PNIPAAm enxertadas nos suportes sofrem uma alteração brusca conformacional, passando do estado colapsado (hidrofóbico) para um estado expandido (hidrofílico), com consequente desadsorção das células devido ao abaixamento de temperatura de apenas alguns graus centígrados. A análise dos materiais revestidos (CYT2, CYT3 e as membranas de PSF/PAN) por microscopia eletrónica de varrimento comprovou a formação de camadas contínuas de hidrogel, revestindo homogeneamente as superfícies dos suportes.

O estudo de superfícies modificadas ou funcionalizadas tem sido feito muitas vezes apenas em estratos 2D, mas os exemplos aqui apresentados mostram que o estudo em dispositivos $3 D$ deverá ser cada vez mais importante. Os materiais desenvolvidos uti- lizando processos que envolvem $\mathrm{CO}_{2}$ supercrítico demonstram claramente a versatilidade desta tecnologia: a possibilidade de produzir desde nano a macro-suportes com elevada pureza, biocompatibilidade e ainda com capacidade de responder a estímulos de temperatura, vai com certeza contribuir de uma forma significativa para atingir o objetivo último de conseguir expandir ex-vivo células estaminais em número que seja clinicamente relevante; o revestimento de membranas com hidrogéis capazes de responder a estímulos externos com grande controlo da morfologia, espessura e densidade do hidrogel constitui também um avanço importante no desenvolvimento de membranas porosas, com capacidade de responder a variações de estímulos externos, para processos avançados de separação e purificação de biomoléculas. Facilmente se antecipa que as tecnologias apresentadas podem ser facilmente usadas numa diversidade de materiais suporte e para revestimentos poliméricos diversos com inúmeras aplicações potenciais no desenvolvimento de dispositivos de microfluidica que respondam a estímulos, em plataformas programáveis de libertação controlada de fármacos, em medicina regenerativa e biotecnologia.
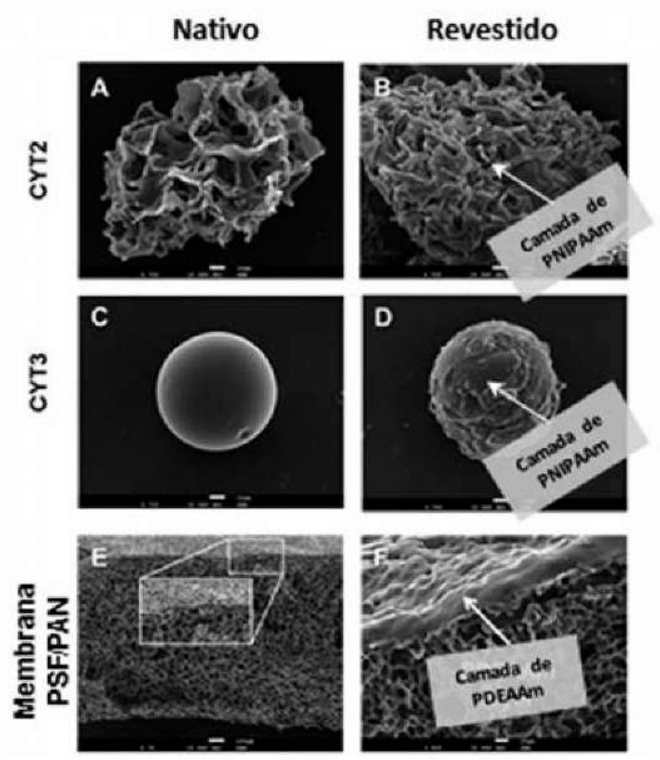

Figura 10 - Imagens dos micro-suportes, antes e depois do processo de revestimento com os hidrogéis, obtidas por microscopia eletrónica de varrimento: (a) CYT2 nativo; (b) CYT2 revestido com PNIPAAm; (c) CYT3 nativo; (d) CYT3 revestido com PNIPAAm; (e) membrana nativa de PSF/PAN com uma região amplificada para melhor visualização dos microporos existentes na superfície de topo da membrana; (f) membrana de PSF/PAN revestida com uma densa camada de PDEAAm obstruindo completamente os poros da superfície. Adaptado (com permissão) de [22]. Copyright (2012) American Chemical Society 
Nos últimos anos assistimos ao estabelecimento de novas estratégias mais sustentáveis de sintese e processamento de polímeros que se revelam capazes de minimizar o número de solventes que se usam num processo químico, ao mesmo tempo que se apresentam como tendo uma variedade de ferramentas interdisciplinares que permitirão o desenvolvimento desde nano- a macroestruturas com uma enorme variedade de aplicações. A verdadeira afirmação destas tecnologias só acontecerá com a demonstração da sua viabilidade técnica e económica à escala industrial.

\section{Agradecimentos}

\section{À Fundação para a Ciência e a Tecno-} logia, pelo financiamento dos projetos de investigação para desenvolvimento de sistemas inteligentes MIT-Pt/BS-CTRM/0051/2008, desenvolvimento de partículas inteligentes para inalação PTDC/EQU-EQU/116097/2009, e bolsas de doutoramento:

SFRH/BD/35382/2007 (E. Costa), SFRH/BD/62475/2009 (T. Barroso), SFRH/BD/75094/2010 (M. Coelho), SFRH/BD/74730/2010 (V. Correia), SFRH/BD/51188/2010 (T. Reis), SFRH/BD/16908/2004 (M. Temtem), SFRH/BPD/38014/2007 (V. Raje), ao Programa MIT-Portugal (Área de Sistemas de Bioengenharia), FEDER e FSE, à Fundação Calouste Gulbenkian pelo financiamento do equipamento de Plasma, ao Laboratório de Análises REQUIMTE e a todos aqueles que têm contribuído para o desenvolvimento destes mesmos projetos.

\section{REFERÊNCIAS}

[1] J. Kost, R. Langer, Responsive polymeric delivery systems, Advanced Drug Delivery Review 46 (2001) 125148.

[2] P. Gupta, K. Vermani, S. Garg, Hydrogels: from controlled release to $\mathrm{pH}$-responsive drug delivery. Drug Discovery Today 7 (2002) 569-579.

[3] B. Jeong, A. Gutowska, Lessons from nature: stimuli responsive polymers and their biomedical applications. Trends Biotechnology 20 (2002) 305311.

[4] M.S. Shim, Y.J. Kwon, Stimuli-responsive polymers and nanomaterials for gene delivery and imaging applications, Advanced Drug Delivery Review (2012), doi:10.1016/j. addr.2012.01.018.

[5] L.Y. Galaev, B. Mattiasson, 'Smart' polymers and what they could do in biotechnology and medicine. Trends Biotechnology 17 (2000) 335-340.

[6] J. Kobayashi, A. Kikuchi, K. Sakai, T. Okano, Aqueous chromatography utilizing hydrophobicity-modified anionic temperature-responsive hydrogel for stationary phases. Journal of Chromatography A 958 (2002) 109-119.

[7] E. Costa, Bioactive beads for local sensing of proteases in 3D engineered tissues. Dissertação de doutoramento, FCT/UNL, Lisboa, 2009.

[8] Y. Kaneko, R. Yoshida, K. Sakai, Y. Sakurai, T. Okano, Temperatureresponsive shrinking kinetics of poly (N-isopropylacrylamide) copolymer gels with hydrophilic and hydrophobic co-monomers. Journal of Membrane Science 101 (1995) 13-22.

[9] (a) P.T. Anastas, J.C. Warner, Green Chemistry: Theory and Practice, Oxford University Press, 2000. (b) V.K. Ahluwalia, M. Kidwai, New Trends in Green Chemistry, Springer, 2004. (c) F.M. Kerton, Alternative Solvents for Green Chemistry, Eds J.H. Clark, G.A. Kraus, RSC Publishing, Cambridge, UK, 2009

[10] M. Temtem, T. Casimiro, J.F. Mano, A. Aguiar-Ricardo, Green Chemistry 9 (2007) $75-79$

[11] T. Casimiro, A.M. Banet-Osuna A.M. Ramos, M. Nunes da Ponte, A. Aguiar-Ricardo, Synthesis of Hghly Cross-Linked Poly(Diethylene Glycol Dimethacrylate) Microparticles in Supercritical Carbon Dioxide. European Polymer Journal 41 (2005) 1947-1953.

[12] T. Casimiro, A. Shariati, C.J. Peters M. Nunes da Ponte, A. Aguiar-Ricardo, Phase Behavior Studies of a Perfluoropolyether in High-Pressure Carbon Dioxide. Fluid Phase Equilibria 224 (2004) 257-261

[13] E. Costa, J. de-Carvalho, T. Casimiro, C. Lobato da Silva, M. Teresa Cidade, A. Aguiar-Ricardo, Tailoring thermoresponsive microbeads in supercritical carbon dioxide for biomedical applications, Journal of Supercritical Fluids 56 (2011) 292-298.

[14] E. Costa, M. Coelho, L.M. Ilharco, A. Aguiar-Ricardo, P.T. Hammond, Tannic Acid Mediated Suppression of PNIPAAm Microgels Thermoresponsive Behavior, Macromolecules 44 (2011) 612-621
[15] E. Costa, M. Lloyd, C. Chopko, A. Aguiar-Ricardo, P. Hammond, Tuning Smart Microgel Swelling and Responsive Behavior through Strong and Weak Polyelectrolyte Pair Assembly, Langmuir 28 (2012) 10082-10090.

[16] M. Temtem, Development of biocompatible and "smart" porous structures using $\mathrm{CO}_{2}$-assisted processes. Tese de doutoramento, FCT/UNL, Lisboa, 2009. Web: http://run.unl.pt/bitstream/10362/1978/1/ Temtem_2009.pdf.

[17] M. Temtem, T. Barroso, T. Casimiro, J.F. Mano, A. Aguiar-Ricardo, Dual stimuli responsive poly( $\mathrm{N}$-isopropylacrylamide) coated chitosan scaffolds for controlled release prepared from a non-residue technology, Journal of Supercritical Fluids 66 (2011) 398404

[18] M. Temtem, D. Pompeu, T. Barroso, J. Fernandes, P.C. Simões, T. Casimiro, A.M. Botelho do Rego, A. AguiarRicardo, Development and characterization of a thermoresponsive polysulfone membrane using an environmental friendly technology, Green Chemistry 11 (2009) 638-645.

[19] D. Wandera, S.R. Wickramasinghe, S.M. Husson, Stimuli-responsive membranes, Journal of Membrane Science 357 (2010) 6-35.

[20] T. Barroso, R. Viveiros, M. Coelho, T. Casimiro, A. Bothelho do Rego, A. Aguiar-Ricardo, "Influence of poly $(\mathrm{N}$ isopropylacrylamide) and poly $\left(\mathrm{N}, \mathrm{N}^{\prime}-\right.$ diethyl acrylamide) coatings on polysulfone/polyacrylonitrile-based membranes for protein separation", Polymer Advanced Technologies (2011), doi:10.1002/pat.2057.

[21] T. Desmet, R. Morent, N. De Geyter, C. Leys, E. Schacht, P. Dubruel, Nonthermal Plasma Technology as a Versatile Strategy for Polymeric Biomaterials Surface Modification: A Review. Biomacromolecules 10(2009) 2351-2378

[22] T. Barroso, R. Viveiros, M. Temtem, T. Casimiro, A. Bothelho do Rego, A. Aguiar-Ricardo, A combined strategy to surface-graft stimuli-responsive hydrogels using plasma activation and supercritical carbon dioxide, ACS Macro Letters 1 (2012) 356-360.

[23] A.K.-L. Chen, X. Chen, A.B.H. Choo, S. Reuveny, S.K.W. Oh, Stem Cell Research 7 (2011) 97-111.

[24] Y. Nie, V.D.J. Bergendahl, J.M. Hei, S.M. Jones, P. Palecek, Biotechnology Progress 25 (2009) 20-31. 
